\title{
A Two-phase Constitutive Model with Damage for Tungsten Heavy Alloy
}

\author{
J J Tang', Z F Liang'*, Y W Zhang ${ }^{2}$ \\ 1. Xi'an Modern Chemistry Research Institute, Xi'an, 710065, \\ China \\ 2. National University of Defense Technology, Changsha, \\ 410073, China
}

\begin{abstract}
Tungsten heavy alloy is a kind of two-phase alloy composed of highstrength tungsten phase and binder phase with good-plasticity. Its mechanical properties and damage evolution are affected by both phases. The static and dynamic mechanical tests of 92.5W-4.9Ni-2.1Fe-0.5Co are carried out. According to the characteristics of stress-strain curves, $\mathrm{KHL}$ model is selected as the constitutive model of the material. Then, combined with Eshelby equivalent inclusion theory and Mori Tanaka mean field theory, the stress-strain relationship of each phase in tungsten heavy alloy is studied by introducing representative volume element (RVE). The damage evolution model considering the strain rate effect is proposed, and according to the different loading states, a two-phase constitutive model with damage is established. By compiling the UMAT subroutine, the test conditions of different strain rates are calculated in ABAQUS software. The simulation results are in good agreement with the test results, and the validity of the proposed model is verified.
\end{abstract}

\section{INTRODUCTION}

Tungsten heavy alloy is a kind of alloy based on tungsten and combined with nickel, copper, iron, manganese, cobalt, chromium and other elements [1]. Generally, the mass fraction of tungsten phase is between $88 \%$ and $97 \%$ [2]. The microstructure of tungsten heavy alloy is a typical two-phase metal structure, which is composed of numerous tungsten grains and the binder phase. The mechanical properties of the two phases are significantly different. The tungsten phase has high strength but poor plasticity. The brittle property limits the application of the pure tungsten. Although the strength of the alloy with binder phase is lower than that of the pure tungsten, the plasticity of the alloy increases obviously, which strengthens the comprehensive properties of the tungsten heavy alloy. It shows that the mechanical properties of tungsten heavy alloy are determined by the mechanical properties of the two phases, and also affected by the strength of the interface between the tungsten grain and the tungsten grain and between the tungsten grain and the binder phase [3][4]. Many research show that tungsten heavy alloy has excellent mechanical properties such as high density, high strength, good toughness, high temperature resistance and impact resistance [5]. Especially under the condition of high-speed impact, it can maintain good penetration ability and lethality.

"Corresponding Author: hotnnncold@163.com 
Therefore, it is widely used as the killing element of weapons, such as the fragment material for killing warhead, core of the armor piercing projectile, etc., which has been widely used in military field [6].

Considering that the loading conditions of tungsten heavy alloy are mostly high strain rate loads, it is particularly important to study the dynamic mechanical properties of materials. At present, there are mainly several kinds of dynamic constitutive models based on different theoretical basis: 1) the empirical model obtained by fitting test data has no clear physical meanings, and the strain rate effect and temperature effect are decoupled. The representative models are Johnson-Cook model (JC model) [7] and KHL model [8][9]; 2) The model based on the micro deformation mechanism of materials has the certain physical theoretical basis. The representative models are Zerilli-Amstrong model (Z-A model) [10][11][12] and Mechanical Threshold Stress model (MTS model) [13][14]; 3) Under the loading condition of high pressure and high strain rate (above $105 \mathrm{~s}^{-1}$ ), assuming that the strain rate effect is saturated, the effect of strain rate on flow stress can be ignored. Therefore, this kind of model is expressed as a function of pressure and temperature, and the representative models are Steinberg-Guinan model (SG model) [15], etc. Although many researchers have proposed various constitutive models based on the understanding of the mechanical behavior of metal materials, considering the feasibility of parameter fitting and engineering application, the empirical fitting model represented by JC model has been widely used. It is easy to modify the original JC model according to different material properties and all the parameters are easy to fit, so it can be applicable for the dynamic deformation behavior of a large number of metal materials. Based on JC model, the coupling of strain hardening, and strain rate effect is taken into account in KHL model, which is suitable for more general stress-strain behaviors.

In addition, the microstructure of tungsten heavy alloy is a typical two-phase structure. The overall mechanical properties of the alloy are closely related to the mechanical properties of the two phases and the bonding strength of the interface. During loading, especially under high strain rate loading such as explosion impact, the stress cannot be rapidly diffused and redistributed between the two phases due to the short loading time, resulting in the difference of stress and strain state between the two phases. In this process, the damage and its evolution process will significantly weaken the mechanical properties of materials. It's known that the fracture modes of tungsten heavy alloy are as follows: cleavage fracture of tungsten grains, ductile fracture of binder phase, separation of tungsten-tungsten interface and separation of tungsten-binder interface [16]. So, the overall mechanical properties of the alloy are closely related to the mechanical properties of the two phases and the bonding strength of the interface. At present, the common dynamic constitutive models such as JC model seldom consider the damage evolution process of materials, and it considers that when the strain reaches the critical value, fracture occurs instantaneously, which is also inconsistent with the true mechanical behavior of materials [17]. In fact, the damage in materials evolves and accumulates gradually. The results show that the plastic deformation behavior of tungsten grains and binder phase in tungsten heavy alloy is different under external load, and continuous damage mechanics [18] considers that the damage evolution is related to the plastic deformation of materials, which leads to the difference of damage evolution between the two phases. In this case, it is unreasonable to ignore the damage effect of two phases and to describe the damage only by the critical fracture parameter. 
Considering that most of the current constitutive models only focus on the mechanical properties of the whole material from the macro scale, there is lack of research on the damage and its evolution process, which cannot fully represent the difference of the two-phase damage evolution and the influence on the overall damage of the alloy, so it still exist many inaccuracies when applied to some loading conditions. In this paper, the influence of the properties and damage evolutions of each phase on the mechanical properties of tungsten heavy alloy are considered. Based on micromechanics theory, the KHL constitutive model with more general applicability is used in this study. Combined with Eshelby equivalent inclusion theory [19] and Mori-Tanaka mean field theory [20], the representative volume element (RVE) and a rate-form damage evolution model [21] are introduced to study the deformation behavior and damage evolution process of tungsten grains and binder phase respectively, and finally, a new two-phase constitutive model of tungsten heavy alloy with damage is established. The simulation results obtained by ABAQUS finite element software are in good agreement with the test results.

\section{TEST WORK}

\subsection{Material and tests}

92.5W-4.9Ni-2.1Fe-0.5Co (wt\%) is prepared in the experiment. Many studies [22][23] show that the strength and plasticity of tungsten heavy alloy can be improved by adding the Co element. The powder parameters are listed in Table 1 . The powder is mixed into the ball mill in proportion for $48 \mathrm{~h}$. The well-mixed powder is added into a specific mold and pressed at $300 \mathrm{MPa}$ for $20 \mathrm{~min}$ for cold isostatic pressing, and then liquid phase sintering is carried out. The sintering temperature is 1460 and is kept for $2 \mathrm{~h}$. The sintering atmosphere is hydrogen. The density is measured by Archimedes method. The geometries of static and dynamic tensile standard specimen are shown in Figure 1, which are obtained by wire-electrode cutting from original samples.

Table 1: Characteristics of powders

\begin{tabular}{lllll}
\hline Powders & W & Ni & Fe & Co \\
\hline Particle size $/ \mu \mathrm{m}$ & $9 \pm 0.3$ & $3 \pm 0.5$ & $7 \pm 0.7$ & $4 \pm 0.5$ \\
Purity $/ \%$ & 99.9 & 99.5 & 99.5 & 99.7 \\
Density $/\left(\mathrm{g} / \mathrm{cm}^{3}\right)$ & 19.32 & 8.91 & 7.8 & 8.9 \\
\hline
\end{tabular}

The quasi-static tensile test is carried out by universal material testing machine at room temperature (298K). The dynamic tensile test system is split Hopkinson tensile bar. The schematic diagram is shown in Figure 2. Its working principle is [24]: the bullet impacts the right end of the incident bar at a speed driven by high pressure gas and forms a direct incident tensile wave in the incident bar. The strain $\varepsilon_{i}$ is recorded by the strain gauge. The reflected stress wave and transmitted stress wave are generated on both ends of the specimen after stretched by incident wave, which are recorded as $\varepsilon_{r}$ and $\varepsilon_{t}$ by strain gauge. Then, according to the strain $\varepsilon_{i}, \varepsilon_{r}$ and $\varepsilon_{t}$ recorded by strain gauge and the elastic wave velocity c, cross-section area $A$ and $A_{0}$ of bar and specimen, elastic modulus $E$ and initial thickness $l_{0}$ of the specimen, the dynamic mechanical properties of the material, including strain rate $\dot{\varepsilon}$, strain $\varepsilon$ and stress $\varepsilon$, can be calculated combining with Equation (2.1) (2.3). 


$$
\begin{gathered}
\dot{\varepsilon}=\frac{-2 c}{l_{0}} \varepsilon_{r} \\
\varepsilon=\frac{-2 c}{l_{0}} \int_{0}^{t} \varepsilon_{r} d t \\
\sigma=\frac{A}{A_{0}} E \varepsilon_{t}
\end{gathered}
$$

In order to obtain better waveforms, the specimen is tied with the incident bar and the transmitted bar by adhesive method. The adhesive is high strength impact resistant adhesive.

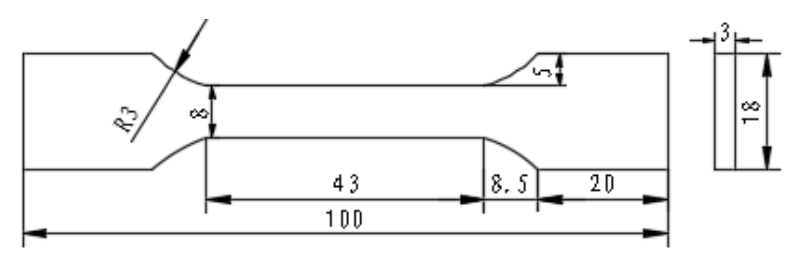

(a)

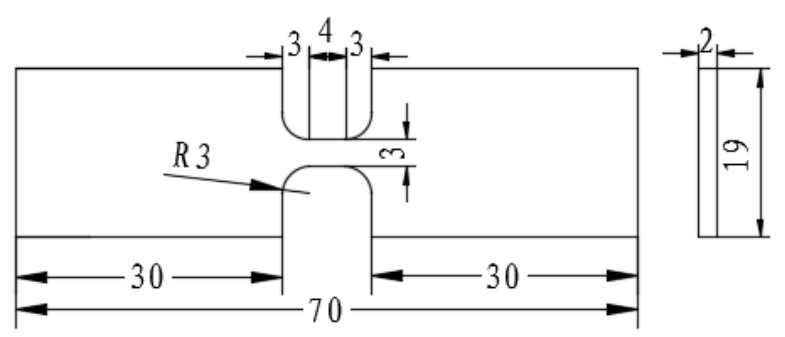

(b)

Figure 1: Schematic of test specimen

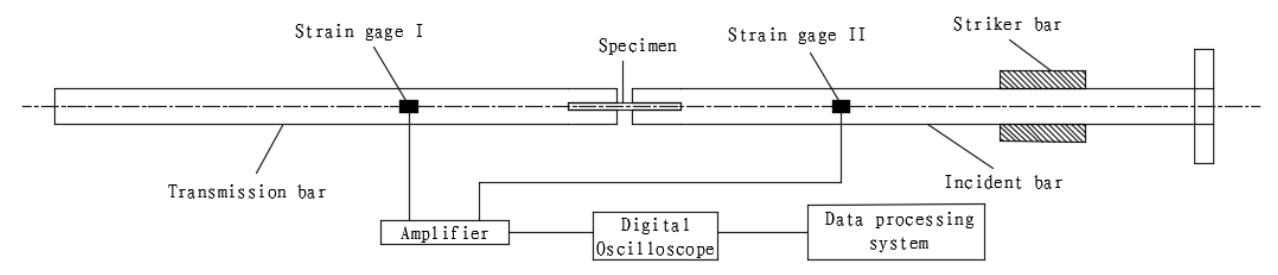

Figure 2: Schematic diagram of split Hopkinson tensile bar 


\subsection{Test results}

According to the quasi-static tensile test data, the mechanical properties of $92.5 \mathrm{~W}-4.9 \mathrm{Ni}-$ 2.1Fe-0.5Co are listed in Table 2. Where, $\rho$ is the density of material, $\sigma_{s}$ is the yield strength, UTS (Ultimate tensile strength) is the ultimate tensile strength, $\delta$ is the elongation percentage of the section, $v$ is Poisson's ratio.

Table 2: Mechanical properties of 92.5W-4.9Ni-2.1Fe-0.5Co

\begin{tabular}{cccccc}
\hline $\boldsymbol{\rho} /\left(\mathbf{g} \cdot \mathbf{c m}^{-3}\right)$ & $\boldsymbol{E} / \mathbf{G P a}$ & $\boldsymbol{\sigma}_{\boldsymbol{s}} / \mathbf{M P a}$ & $\boldsymbol{U T S} / \mathbf{M P a}$ & $\boldsymbol{\delta}$ & $\boldsymbol{v}$ \\
\hline 17.56 & 308 & 661 & 1114 & 0.158 & 0.33 \\
\hline
\end{tabular}

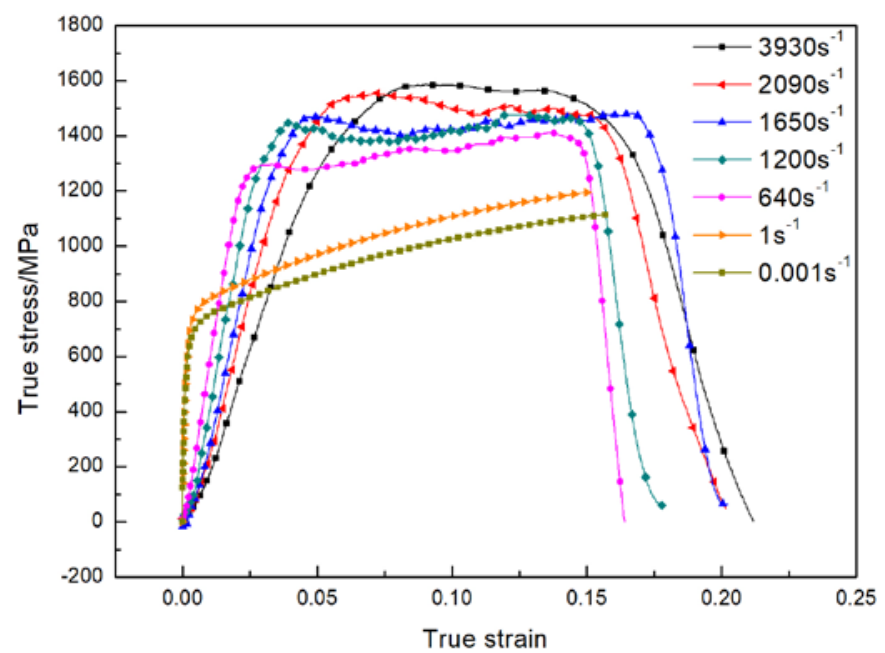

Figure 3: Flow stress-strain curves of 92.5W-4.9Ni-2.1Fe-0.5Co

Figure 3 shows the flow stress-strain curve of 92.5W-4.9Ni-2.1Fe-0.5Co in the range of strain rate $0.001 \mathrm{~s}^{-1} \sim 3930 \mathrm{~s}^{-1}$. It can be seen from the figure that with the increase of strain rate, the flow stress and the dynamic yield strength increases significantly, showing obvious strain rate hardening effect. This is consistent with the results observed in many literatures [25][26]. In addition, under the condition of low strain rate loading $\left(0.001 \mathrm{~s}^{-1}, 1 \mathrm{~s}^{-1}\right.$ and $640 \mathrm{~s}^{-1}$ strain rate), the flow stress has obvious strain hardening phenomenon in the plastic deformation stage, but with the further increase of strain rate, the strain hardening phenomenon is no longer significant, and the trend of stress-strain curve in the plastic deformation stage is relatively gentle. Strain softening even occurs in the case of $2090 \mathrm{~s}^{-1}$ and $3930 \mathrm{~s}^{-1}$ strain rates.

Since the JC model implies that the stress-strain curve trend of the material under different strain rates should be similar, which is inconsistent with the characteristics of dynamic tensile stress-strain curve of $92.5 \% \mathrm{~W}$ tungsten alloy in this paper, a more general KHL constitutive model is selected to describe the macroscopic stress-strain characteristics of the material. 


\section{TWO-PHASE CONSTITUTIVE MODEL OF TUNGSTEN HEAVY ALLOY WITH DAMAGE}

\subsection{KHL constitutive mode}

The form of KHL constitutive model is similar to JC model. The effects of strain rate, temperature and plastic deformation are considered when describing the plastic flow stress of materials. Compared with the three decoupling forms of JC model, KHL model also considers the effect of strain rate in the strain term, so it applies to more materials. Moreover, according to the tensile test results of 92.5\%W tungsten heavy alloy shown in Figure 3, KHL model can describe the stress-strain relationship more appropriately than JC model. It takes the form of

$$
\sigma=\left[A+B\left(1-\frac{\ln \dot{\varepsilon}}{\ln D_{0}}\right)^{n_{1}} \varepsilon^{n_{0}}\right]\left(\frac{\dot{\varepsilon}}{\dot{\varepsilon}_{0}}\right)^{C}\left(\frac{T_{m}-T}{T_{m}-T_{r}}\right)^{m}
$$

where, $D_{0}$ is the upper limit of the reference strain rate, here $D_{0}=10^{6} \mathrm{~s}^{-1}, T_{m}$ is the melting point of the material (the melting point of tungsten heavy alloy is usually $1730 \mathrm{~K}$ ), $T_{r}=296 \mathrm{~K}$ is the reference temperature, $A, B, n_{0}, n_{1}, C$ and $m$ are material parameters obtained by fitting test data.

\subsection{Establish stress-strain relation of each phase}

According to the results of micro-morphology analysis, tungsten heavy alloy is mainly composed of spherical reinforced $\mathrm{W}$ phase and Ni-Fe-W binder phase. The mechanical properties of tungsten heavy alloy are determined by the mechanical properties of the two phases, and also affected by the interaction between the two phases, such as the interfacial strength of W-binder phase. Meanwhile, the damage of tungsten heavy alloy is also affected by the damage of the $\mathrm{W}$ phase and binder phase [27].

In order to study the effect of mechanical properties and interaction of two phases on the overall properties of the material, an equivalent medium with the same elastic properties is introduced to characterize the composite. Here, the representative volume element (RVE) in continuum mechanics is introduced as the study object. The binder phase and tungsten phase are regarded as the matrix phase and reinforced W phase of RVE. According to Eshelby equivalent inclusion theory and Mori-Tanaka mean field theory, the stress-strain relationship between RVE and two phases is established.

As shown in Figure 4, the three curves represent the stress-strain curves of reinforced W phase, RVE and matrix phase respectively from top to bottom. The subscript m represents the matrix phase and w represents the reinforced $\mathrm{W}$ phase. The elastic modulus of RVE is defined as and the initial static yield strength is $\sigma_{y}$. The corresponding strain is $\varepsilon_{y}$. The stress-strain curve of RVE can be obtained directly by test, and the elastic modulus of reinforced $\mathrm{W}$ phase is $E_{w}$. The initial static yield strength is $\sigma_{y w}$, and the corresponding strain is $\varepsilon_{y w}$. Considering that the reinforced $\mathrm{W}$ phase accounts for the majority of $92.5 \%$ tungsten heavy alloy, it can be reasonably considered that the hardening law of reinforced $\mathrm{W}$ phase is similar to that of tungsten heavy alloy, and also conforms to the law of nonlinear hardening, which is consistent with the research results [28]; the elastic modulus of matrix phase is $E_{m}$. The initial static yield strength is $\sigma_{y m}$, and the corresponding strain is $\varepsilon_{y m}$. 
Because of the small proportion of matrix phase, the influence of hardening behavior on RVE is weaker than that of reinforced $\mathrm{W}$ phase. Considering that the real plastic behavior of matrix phase is difficult to be directly characterized by test results, in order to simplify the model, it is assumed that the constitutive behavior of matrix phase also conforms to the nonlinear hardening law.

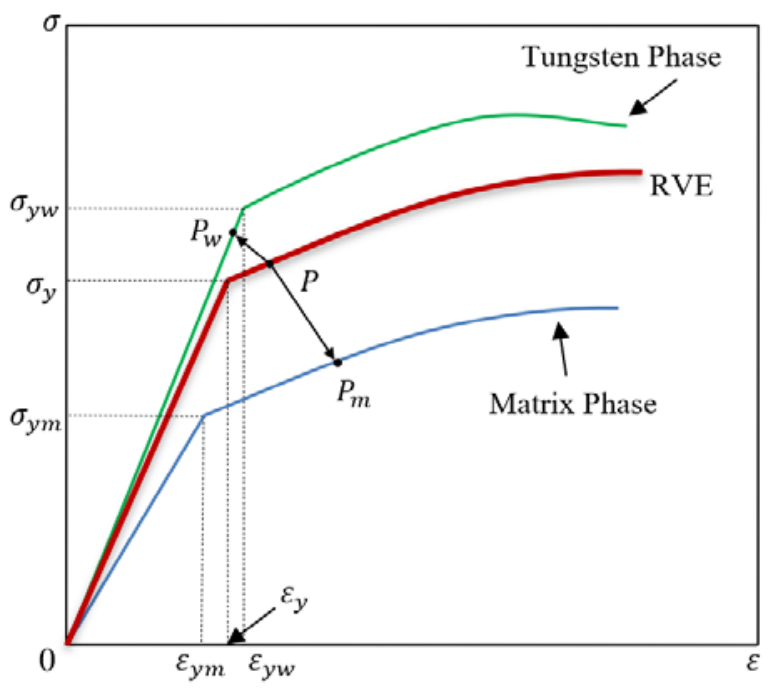

Figure 4: Schematic diagram of stress-strain curves of each phase

According to Eshelby's equivalent inclusion theory, the stress in the matrix and reinforced $\mathrm{W}$ phase can be expressed respectively as

$$
\begin{gathered}
\sigma_{m}=E_{m} \varepsilon_{m}=E_{m}(\bar{\varepsilon}+\tilde{\varepsilon}) \\
\sigma_{w}=E_{w} \varepsilon_{w}=E_{w}\left(\bar{\varepsilon}+\tilde{\varepsilon}+\varepsilon_{w}^{p t}\right)=E_{m}\left(\bar{\varepsilon}+\tilde{\varepsilon}+\varepsilon_{w}^{p t}-\varepsilon_{w}^{*}\right)
\end{gathered}
$$

where, $\sigma_{m}, \varepsilon_{m}$ and $\sigma_{w}, \varepsilon_{w}$ are the mean stress and strain in matrix phase and reinforced $\mathrm{W}$ phase respectively. $\bar{\varepsilon}$ is the strain of RVE, and $\tilde{\varepsilon}$ is the disturbance strain in two phases due to the existence of the reinforced phase. $\varepsilon_{w}^{p t}$ is the additional disturbance strain caused by the presence of reinforced phase, which is also known as the perturbation strain. $\varepsilon_{w}^{*}$ is the eigen strain in the reinforced phase. Eshelby considered that the reinforced phase can be regarded as the equivalent reinforced phase with eigen strain and the same stiffness tensor as the matrix phase and satisfy the relationship. 


$$
\varepsilon_{w}^{p t}=S \varepsilon_{w}^{*}
$$

where, $S$ is the fourth order Eshelby tensor, which is related to Poisson's ratio of matrix phase and shape of reinforced phase and indicates the influence of inhomogeneity of reinforced phase. For the isotropic case, the Eshelby tensor is expressed as

$$
S=\left[\begin{array}{cccccc}
S_{1111} & S_{1122} & S_{1133} & 0 & 0 & 0 \\
S_{2211} & S_{2222} & S_{2233} & 0 & 0 & 0 \\
S_{3311} & S_{3322} & S_{3333} & 0 & 0 & 0 \\
0 & 0 & 0 & S_{1212} & 0 & 0 \\
0 & 0 & 0 & 0 & S_{2323} & 0 \\
0 & 0 & 0 & 0 & 0 & S_{3131}
\end{array}\right]
$$

When the shape of reinforced $\mathrm{W}$ phase is approximately regarded as a sphere, $S$ will be simplified as

$$
\begin{gathered}
S_{1111}=S_{2222}=S_{3333}=\frac{7-5 v}{15(1-v)} \\
S_{1122}=S_{2233}=S_{3311}=\frac{1-5 v}{15(1-v)} \\
S_{1212}=S_{2323}=S_{3131}=\frac{4-5 v}{15(1-v)}
\end{gathered}
$$

where, $v$ is the Poisson's ratio of matrix phase. According to the Equation (3.2) and (3.3), there are

$$
\begin{gathered}
\varepsilon_{w}=\varepsilon_{m}+\varepsilon_{w}^{p t} \\
E_{w} \varepsilon_{w}=E_{m}\left(\varepsilon_{w}-\varepsilon_{w}^{*}\right)
\end{gathered}
$$

Substituting Equation (3.10) into Equation (3.4), there is

$$
\varepsilon_{w}^{p t}=S E_{m}^{-1}\left(E_{m}-E_{w}\right) \varepsilon_{w}
$$

Combining with Equation (3.9), the relationship between the average strain of matrix phase and reinforced phase is expressed as

$$
\varepsilon_{w}^{p t}=S E_{m}^{-1}\left(E_{m}-E_{w}\right) \varepsilon_{w}
$$


Define the strain tensor $A_{r}$ as

$$
A_{r}=\left[I-S E_{m}^{-1}\left(E_{m}-E_{r}\right)\right]^{-1}
$$

where,

$$
\begin{gathered}
A_{m}=I \\
A_{w}=\left[I-S E_{m}^{-1}\left(E_{m}-E_{w}\right)\right]^{-1}
\end{gathered}
$$

Then, Equation (3.12) can be expressed as

$$
\varepsilon_{w}=A_{w} \varepsilon_{m}
$$

According to the average rule of stress and strain, the stress-strain relationship of RVE and each phase can be expressed as

$$
\begin{aligned}
& \sigma=f_{m} \sigma_{m}+f_{w} \sigma_{w} \\
& \varepsilon=f_{m} \varepsilon_{m}+f_{w} \varepsilon_{w}
\end{aligned}
$$

where, $\sigma$ and $\varepsilon$ are the total mean stress and mean strain of RVE, $f_{m}$ and $f_{w}$ are the volume fraction of matrix phase and reinforced phase respectively, which satisfy the following equation:

$$
f_{m}+f_{w}=1
$$

Substituting Equation (3.16) into Equation (3.17) and (3.18), there are

$$
\begin{gathered}
\sigma=f_{m} \sigma_{m}+f_{w} E_{w} A_{w} E_{m}^{-1} \sigma_{m}=\left(f_{m}+f_{w} E_{w} A_{w} E_{m}^{-1}\right) \sigma_{m} \\
\varepsilon=f_{m} \varepsilon_{m}+f_{w} A_{w} \varepsilon_{m}=\left(f_{m}+f_{w} A_{w}\right) \varepsilon_{m}
\end{gathered}
$$

The relationship between the mean stress and strain of each phase and the total mean stress and strain of RVE can be obtained 


$$
\begin{gathered}
\sigma_{m}=E_{m}\left(f_{m} E_{m}+f_{w} E_{w} A_{w}\right)^{-1} \sigma \\
\sigma_{w}=E_{w} A_{w}\left(f_{m} E_{m}+f_{w} E_{w} A_{w}\right)^{-1} \sigma \\
\varepsilon_{m}=\left(f_{m}+f_{w} A_{w}\right)^{-1} \varepsilon \\
\varepsilon_{w}=A_{w}\left(f_{m}+f_{w} A_{w}\right)^{-1} \varepsilon
\end{gathered}
$$

As shown in Figure 4, when the RVE is in the stress-strain state of point $P$, the stress-strain states of matrix phase and reinforced $\mathrm{W}$ phase can be calculated as $P_{m}$ and $P_{w}$ by Equation (3.23) and (3.24).

The constitutive relation of RVE in elastic deformation is expressed as

$$
\sigma=E \varepsilon
$$

According to Equation (3.20) and (3.21), the relationship between elastic modulus $E$ of RVE and elastic modulus of matrix phase and reinforced W phase can be expressed as follows:

$$
E=\left(\sum_{r=m, w} f_{r} A_{r} E_{r}\right)\left(\sum_{r=m, w} f_{r} A_{r}\right)^{-1}=\frac{f_{m} E_{m}+f_{w} A_{w} E_{w}}{f_{m}+f_{w} A_{w}}
$$

For 92.5W-4.9Ni-2.1Fe-0.5Co, the elastic modulus E of RVE can be determined by test, which has been listed in Table 2 . The elastic modulus $E_{w}$ of W phase is obtained by consulting the literature [28]. So, the elastic modulus $E_{m}$ of the matrix phase can be calculated by Equation (3.25).

\subsection{Establish damage evolution model}

\subsubsection{Damage variable definition}

In general, the initiation and growth of micropores and microcracks are defined as damage and its evolution [29]. However, it is still difficult to accurately measure and monitor the development of voids and cracks on the microscale by means of current technology. Therefore, damage is introduced from the perspective of phenomenology in continuum damage mechanics. The damage is regarded as an internal defect with uniform and continuous distribution in RVE, which can be described by homogenization variables. For isotropic materials, the damage variable degenerates to scalar. Two methods are usually used to describe the damage state of materials: (1) reduction of effective stress area; (2) reduction of effective elastic modulus. 


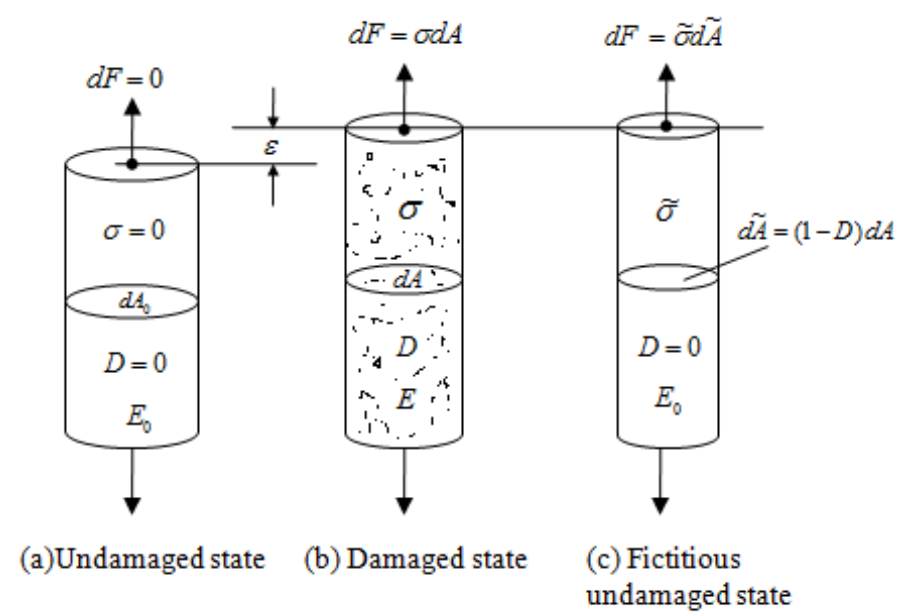

Figure 5: Diagram of damaged rod under uniaxial tension

When the effective elastic modulus is used to describe the damage state, the uniaxial tension condition of a rod is taken as an example. Figure 5(a) is the original state of the material, and Figure 5(b) and 5(c) are the damaged state and the fictitious undamaged state of the material respectively. According to the principle of strain equivalence, the elastic strain $\varepsilon$ caused by the effective stress $\tilde{\sigma}$ in Figure 5(c) should be the same as that caused by stress $\sigma$ in Figure 5(b):

$$
\tilde{\sigma}=E_{0} \varepsilon, \sigma=E(D) \varepsilon
$$

or

$$
\varepsilon=\frac{\sigma}{E(D)}=\frac{\widetilde{\sigma}}{E_{0}}
$$

where, $E_{0}$ and $E(D)$ are the elastic modulus of the material in the initial undamaged state and the damaged elastic modulus, respectively. The effective stress can be defined as

$$
\tilde{\sigma}=\frac{E_{0}}{E(D)} \sigma
$$

Then the relationship between damage state and elastic modulus is

$$
E(D)=(1-D) E_{0}
$$

or

$$
D=1-\frac{E(D)}{E_{0}}
$$




\subsubsection{Establish damage evolution model}

It is generally believed that the microscopic essence of plastic flow behavior of metal materials is the irreversible dislocation motion based on thermo-activation mechanism. Moreover, the dynamic constitutive behavior of materials is also related to strain rate. For the plastic deformation behavior within strain rate, the strain rate and temperature usually satisfy Arrhenius equation [30]:

$$
\dot{\varepsilon}=\dot{\varepsilon}_{0} \exp \left(-\frac{U_{S}(\sigma, T)}{k T}\right)
$$

where, $\dot{\varepsilon}$ is the strain rate, $\dot{\varepsilon}$ is the rate factor, also known as the nominal limit strain rate, which is affected by the density of movable dislocations. $U_{S}$ is the thermal activation energy, which is related to the stress $\sigma$ and temperature $T$, and $k$ is the Boltzman constant. According to continuum damage mechanics, the damage of metal materials is caused by plastic deformation. Compared with Equation (3.31), the evolution of damage can also be regarded as the thermal activation behavior caused by dislocation motion. There is

$$
\dot{D}=\frac{\partial D}{\partial t}=\dot{D}_{0} \exp \left(-\frac{U_{D}(\sigma, T)}{k T}\right)
$$

where, $\dot{D}$ is the damage evolution rate. Similar to $\dot{\varepsilon}, \dot{D}$ is the rate factor of damage evolution, $U_{D}$ is the thermal activation energy related to damage evolution. Suppose that for the same material, the thermal activation energy $U_{S}$ and $U_{D}$ satisfies the proportional relation

$$
U_{D}=a U_{S}
$$

where, a is the material parameter. The relationship between damage evolution rate and strain rate can be obtained from Equation (3.31), (3.32) and (3.33)

$$
\frac{\dot{D}}{\dot{D}_{0}}=\left(\frac{\dot{\varepsilon}}{\dot{\varepsilon}_{0}}\right)^{\mathrm{a}}
$$

or

$$
\dot{D}=K_{D} \dot{\varepsilon}^{a}
$$

Integrating Equation (3.35), the damage evolution equation in the form of rate is obtained

$$
D=K_{D} \Psi_{D}(\dot{\varepsilon})
$$

where, $K_{D}=\dot{D}_{0} / \dot{\varepsilon}_{0}^{a}, \Psi_{D}(\dot{\varepsilon})=\int_{0}^{\mathrm{t}} \dot{\varepsilon}^{a} d t$. 
Besides, there is usually a certain strain threshold value $\varepsilon_{t h}$ for the damage evolution. By integrating Equation (3.35), under the loading condition of constant strain rate, it can be obtained

$$
D=K_{D} \dot{\varepsilon}^{a-1}\left(\varepsilon-\varepsilon_{t h}\right) \quad \varepsilon \geq \varepsilon_{t h}
$$

In addition, the dynamic failure criterion is described by

$$
D \geq D_{C}
$$

where, $D_{C}$ is the critical damage value. It is considered that when the damage value is greater than $D_{C}$, the material will be completely fractured.

If Equation (3.37) is extended to more general cases, the relationship between damage value $D$ and strain $\varepsilon$ may be nonlinear. Hence, the dynamic damage evolution model of materials in general is obtained

$$
D=D_{k}\left(\dot{\varepsilon} / \dot{\varepsilon}_{0}\right)^{a-1}\left(\varepsilon-\varepsilon_{t h}\right)^{b}, \varepsilon \geq \varepsilon_{t h}
$$

where, $D_{k}, a$ and $b$ are material parameters, which can be fitted by test data.

\subsection{Two-phase constitutive model of tungsten heavy alloy with damage}

Combining the damage evolution model with the stress-strain relationship of RVE and each phase obtained in Section 3.2, a two-phase constitutive model of tungsten heavy alloy with damage is established. The damage value of matrix phase is defined as $D_{m}$. The damage value of reinforced W phase is defined as $D_{w}$. The total damage value of RVE is $D$.

According to damage mechanics theory, the damage begins to occur in the plastic stage due to irreversible deformation and is gradually accumulated with the increase of plastic deformation. However, as shown in Figure 4, the mechanical behaviors of matrix phase and reinforced W phase are significantly different. When RVE is loaded, the strain states of matrix phase and reinforced $\mathrm{W}$ phase are different, which makes the damage evolution process and damage value in the two phases inconsistent. During the powder sintering process, the powders of two phases fully mix and interact to form a whole structure for tungsten heavy alloy, which determines that the damage of materials not only evolves independently in each phase, but also is affected by the interaction between each phase. Then, a two-phase constitutive model of tungsten heavy alloy with damage is proposed.

To simplify the analysis, only the uniaxial tensile load is discussed here. With the increase of load on RVE, the damage evolution of two-phase constitutive model under the uniaxial tension can be divided into the following three cases:

The tensile load is small, so the matrix phase, reinforced W phase and RVE are in the elastic stage and no damage occurs. The stress-strain relationship of the whole RVE is described as 


$$
\sigma=E \varepsilon
$$

where, $\sigma, \varepsilon$ and $E$ are the stress, strain and elastic modulus of RVE.

With the increase of load, the matrix phase first occurs to plastic deformation and begins to appear damage due to the low yield strength. RVE and the reinforced $\mathrm{W}$ phase are still in the elastic stage. Then the strain of matrix is obtained by Equation (3.23)

$$
\varepsilon_{m}=\left(f_{m}+f_{w} A_{w}\right)^{-1} \varepsilon
$$

According to Equation (3.39), the damage $D_{m}$ of matrix phase can be calculated by

$$
D_{m}=D_{k}\left(\dot{\varepsilon} / \dot{\varepsilon}_{0}\right)^{a-1}\left(\varepsilon_{m}-\varepsilon_{t h, m}\right)^{b}
$$

where, $\varepsilon_{t h, m}$ is the strain threshold of matrix phase for damage.

According to Equation (3.29), the elastic modulus $E_{D m}$ of matrix phase with damage is updated by

$$
E_{D m}=\left(1-D_{m}\right) E_{m}
$$

In this stage, the reinforced $\mathrm{W}$ phase is still in elastic deformation, which no damage occurs. Therefore, the damaged elastic modulus $E_{D}$ of RVE is updated according to Equation (3.28)

$$
E_{D}=\frac{f_{m} E_{D m}+f_{w} A_{w} E_{w}}{f_{m}+f_{w} A_{w}}
$$

The damage value of RVE is obtained as follows

$$
D=1-\frac{E_{D}}{E}
$$

Then, the stress-strain state of RVE is updated to

$$
\sigma=(1-D) E \varepsilon=E_{D} \varepsilon
$$

When the load is large enough, both the matrix phase and the reinforced $\mathrm{W}$ phase occur to the plastic deformation, but the damages of the two phases are also different due to the different plastic deformation. Therefore, the total strain and plastic strain of each phase are calculated by Equation (3.23) respectively

$$
\left\{\begin{array}{c}
\varepsilon_{m}=\left(f_{m}+f_{m} A_{w}\right)^{-1} \varepsilon \\
\varepsilon_{w}=A_{w}\left(f_{m}+f_{m} A_{w}\right)^{-1} \varepsilon
\end{array}\right.
$$


The damage $D_{m}$ and $D_{w}$ of the matrix phase and the reinforced W phase can be calculated by Equation (3.39)

$$
\left\{\begin{array}{l}
D_{m}=D_{k}\left(\dot{\varepsilon} / \dot{\varepsilon}_{0}\right)^{a-1}\left(\varepsilon_{m}-\varepsilon_{t h, m}\right)^{b} \\
D_{w}=D_{k}\left(\dot{\varepsilon} / \dot{\varepsilon}_{0}\right)^{a-1}\left(\varepsilon_{w}-\varepsilon_{t h, w}\right)^{b}
\end{array}\right.
$$

where, $\varepsilon_{t h, w}$ is the strain threshold of the reinforced $W$ phase for damage. The damage calculation of RVE is the same as that of (2), then the flow stress model of RVE is expressed as

$$
\sigma=(1-D)\left[A+B\left(1-\frac{\ln \dot{\varepsilon}}{\ln D_{0}}\right)^{n_{1}} \varepsilon^{n_{0}}\right]\left(\frac{\dot{\varepsilon}}{\dot{\varepsilon}_{0}}\right)^{C}\left(\frac{T_{m}-T}{T_{m}-T_{r}}\right)^{m}
$$

Based on the realization of microstructure and mechanical properties of tungsten heavy alloy, the two-phase constitutive model with damage is established by analyzing the above three loading conditions.

\section{FINITE ELEMENT SIMULATION}

\subsection{Parameter calibration}

Firstly, the parameters in the constitutive model Equation (3.1) of RVE are calibrated. When the $\mathrm{x}$-axis of the flow stress-strain curve obtained by test is changed to plastic strain without considering the failure section, Figure 3 is replotted to Figure 6. The test in Section 2 is carried out at room temperature without considering the adiabatic temperature rise and environmental temperature variation, so the temperature term is omitted. Equation (3.1) becomes into

$$
\sigma=\left[A+B\left(1-\frac{\ln \dot{\varepsilon}}{\ln D_{0}}\right)^{n_{1}} \varepsilon_{p}^{n_{0}}\right]\left(\frac{\dot{\varepsilon}}{\dot{\varepsilon}_{0}}\right)^{C}
$$

where, the upper limit of the reference strain rate $D_{0}=10^{6} s^{-1}$, the reference strain rate $\dot{\varepsilon}=1 \mathrm{~s}^{-1}$. Under the loading condition of strain rate $1 \mathrm{~s}^{-1}$, Equation (4.1) changes to $\sigma=A+B \varepsilon_{p}^{n_{0}}$. According to the relationship of $\sigma \sim \varepsilon_{p}$, the parameters $A, B$, and $n_{0}$ can be obtained. When $\varepsilon_{p}$ is taken to be 0 , Equation (4.1) is simplified to

$$
\sigma=A\left(\frac{\dot{\varepsilon}}{\dot{\varepsilon}_{0}}\right)^{C}
$$

Then, the parameters of the reinforced $\mathrm{W}$ phase and matrix phase are calibrated. According to Section 3.2, the reinforced $\mathrm{W}$ phase also has a nonlinear hardening trend, and the constitutive model of pure tungsten is as follows 


$$
\sigma=\left[A_{w}+B_{w} \varepsilon_{p}^{n_{w}}\right]\left[1+C_{w} \ln \frac{\dot{\varepsilon}}{\dot{\varepsilon}_{0}}\right]
$$

where, $A_{w}=890 \mathrm{MPa}, B_{w}=144 \mathrm{MPa}, n_{w}=0.5026, C_{w}=0.0745$.

In order to simplify the calculation, it can be reasonably assumed that the matrix phase and RVE have the same strain hardening and strain rate hardening effects and the parameters of constitutive model are the same as RVE except parameter $A_{m}$. Referring to the method of Wan [31] to determine the parameter $A_{m}$, discuss the material parameters in a reasonable range, which are difficult to be determined. Considering the low strength of matrix phase and combining with the results of the finite element simulation, the parameter is determined as $A_{m}=70 \% A$.

Finally, the parameters of damage evolution model are calibrated. Substituting Equation (3.39) into Equation (3.51) without considering the temperature term, there is

$$
\sigma=\left(1-D_{k}\left(\dot{\varepsilon} / \dot{\varepsilon}_{0}\right)^{a-1}\left(\varepsilon-\varepsilon_{t h}\right)^{b}\left[A+B\left(1-\frac{\ln \dot{\varepsilon}}{\ln D_{0}}\right)^{n_{1}} \varepsilon_{p}^{n_{0}}\right]\left(\frac{\dot{\varepsilon}}{\dot{\varepsilon}_{0}}\right)^{C}\right.
$$

When $\dot{\varepsilon}=1 s^{-1}$, it takes to $\sigma=\left(1-D_{k} \varepsilon_{p}^{b}\right)\left(A+B \varepsilon_{p}^{n_{0}}\right)$. According to the $\sigma \sim \varepsilon_{p}$ curve in Figure 6, the parameter $D_{k}$ and $b$ are obtained. By substituting the fitted parameters to the Equation (4.3), the corresponding $a$ of each strain rate $\dot{\varepsilon}$ can be calculated. Then take the average to determine the parameter $a$. For simplicity, the matrix phase and the reinforced $\mathrm{W}$ phase share a set of damage parameters with RVE. $\varepsilon_{t h, m}$ and $\varepsilon_{t h, w}$ is considered as the strain value when the plastic strain of each phase is taken as 0 .

In conclusion, all parameters of the two-phase constitutive model of tungsten heavy alloy with damage are calibrated, and the specific parameters are listed in Table 3.

\begin{tabular}{cc} 
Table 3: Parameters of two-phase constitutive model with damage of $92.5 \mathrm{~W}$ \\
\hline$A / \mathrm{MPa}$ & 713.9 \\
$B / \mathrm{MPa}$ & 1915.9 \\
$n_{0}$ & 0.73 \\
$n_{1}$ & 2.84 \\
$C$ & 0.0809 \\
$A_{w} / \mathrm{MPa}$ & 890 \\
$B_{w} / \mathrm{MPa}$ & 144 \\
$n_{w}$ & 0.5026 \\
$C_{w}$ & 0.0745 \\
$E_{w} / \mathrm{MPa}$ & 413 \\
$A_{m} / \mathrm{MPa}$ & 499.7 \\
$D_{k}$ & 0.2 \\
$a$ & 0.89 \\
$b$ & 0.317 \\
\hline
\end{tabular}




\subsection{Finite element simulation}

Considering the symmetry of the model, the finite element model of a 1/4 split Hopkinson tensile bar and specimen is established in ABAQUS, as shown in Figure 7. The length and radius of incident bar and transmission bar are $970 \mathrm{~mm}$ and $10 \mathrm{~mm}$, respectively. The material model of the bar is elastic. The elastic modulus and Poisson's ratio are 72GPa and 0.33 respectively, and the density is $2.7 \times 10^{3} \mathrm{~kg} / \mathrm{m}^{3}$. Schematic of dynamic specimen is shown in Figure 1(b). Two symmetrical planes are respectively applied symmetry constraints. All contact surfaces of the specimen and the two bars are tie constraints. The element type is C3D8. Stress pulses are applied to the right end of the incident bar to replace the bullet.

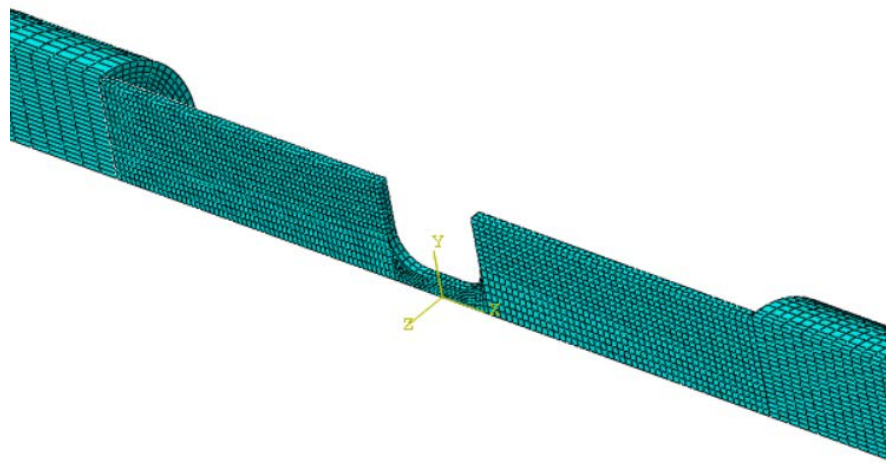

Figure 7: The finite element model of specimen

The maximum time step of incremental step is determined by the stability limit of the element which can be calculated by

$$
\Delta t_{\text {stable }}=\frac{L_{0}}{C}
$$

where, $L_{0}$ is the characteristic length of the element, $C$ is the longitudinal wave velocity of the material. In this model, the characteristic length of elements in bar is $10 \mathrm{~mm}$, and the propagation velocity of stress wave in bar is $C_{d}=5164 \mathrm{~m} / \mathrm{s}$, so the maximum time step is $2 \times 10^{-6} s$.

Considering that the damage evolution is a continuous accumulation process and the stress-strain state needs to be updated according to the previous damage value, the UMAT subroutine is compiled in ABAQUS/standard for the simulation. Implicit stress updating algorithm is referred to the book [32], then the whole split Hopkinson tensile bar test is simulated according to the proposed two-phase constitutive model with damage. The whole simulation flowchart is shown in Figure 8. 


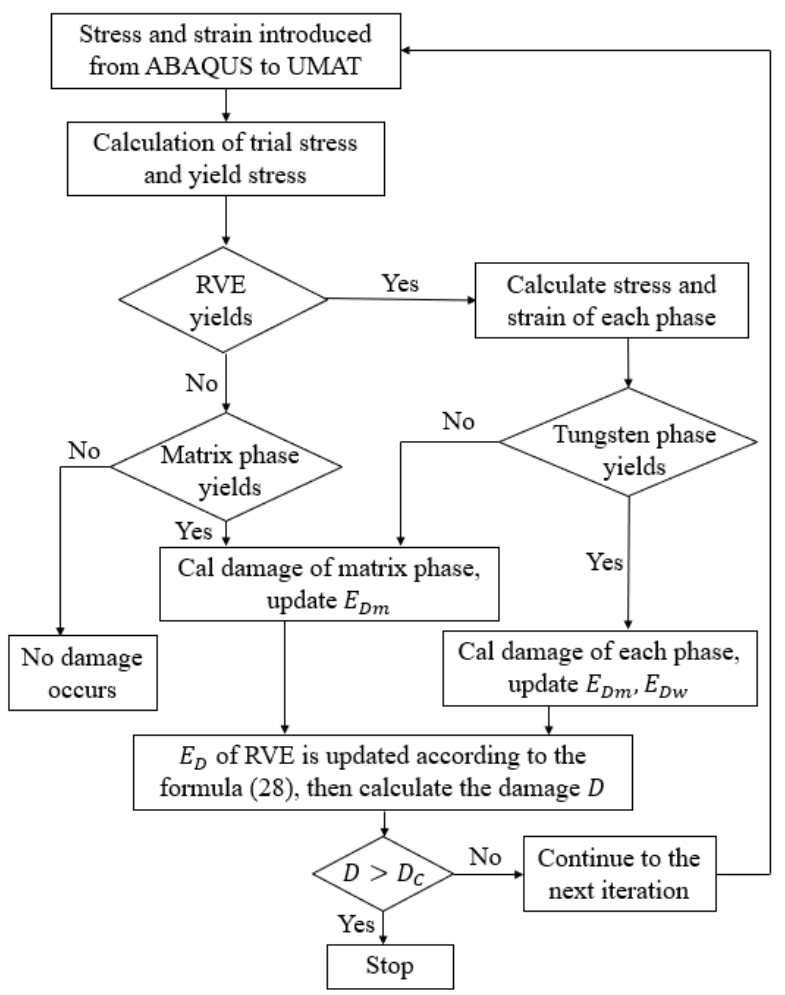

Figure 8: Simulation flowchart for two-phase constitutive model with damage

The simulation results of the specimen under the condition of $\dot{\varepsilon}=1200 \mathrm{~s}^{-1}$ are shown here in Figure 9. Firstly, it can be seen that the front edge of stress wave reaches the specimen at the time $t_{0}=1.96 \times 10^{-4} \mathrm{~s}$, which is consistent with the time calculated by the longitudinal wave velocity. The stress value on the specimen is 0 before $t_{0}$.
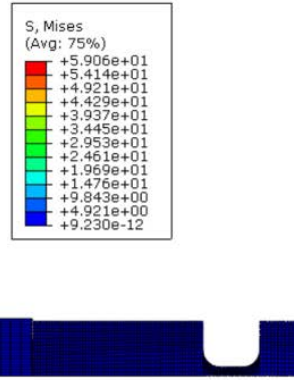

Figure 9: The stress propagation diagram of the specimen at $t_{0}=1.96 \times 10^{-4} \mathrm{~s}$ 
Figure 10 shows the stress distribution diagram of the specimen at $t=3.74 \times 10^{-4} \mathrm{~s}$. After the steady rising stage of stress wave, some of the stress wave continues to propagate in the incident bar through reflection, and some enters into the transmission bar. After multiple reflections of the stress wave, the stress state of the middle section of the specimen tends to be stable and the deformation of each point is uniform. Draw the strain rate-time curve of any point in the middle section. As shown in Figure 11, after the stress rising section, the strain rate gradually adjusts to a stable state. The stress-plastic strain curve is shown in Figure 12. The black solid line represents the test result, and the red dotted line represents the simulation result by the two-phase KHL model. The simulation curve is basically consistent with the test curve, which verifies the effectiveness of the proposed model.

In addition, Figure 12 also compares the simulation result of JC model under strain rate $1200 \mathrm{~s}^{-1}$ with that of the proposed model, and JC model has the obvious inaccuracy, which is shown as the red dashed line. The static stress-strain curve of $92.5 \%$ tungsten heavy alloy satisfies the strain hardening effect, while the strain hardening effect under dynamic tensile condition is not obvious and even strain softening phenomenon occurs. However, JC model is only applicable to the materials with similar stress-strain curves at different strain rates, which is inconsistent with the tensile mechanical properties of $92.5 \%$ tungsten heavy alloy, so it is not suitable to describe the dynamic tensile properties of $92.5 \%$ tungsten heavy alloy

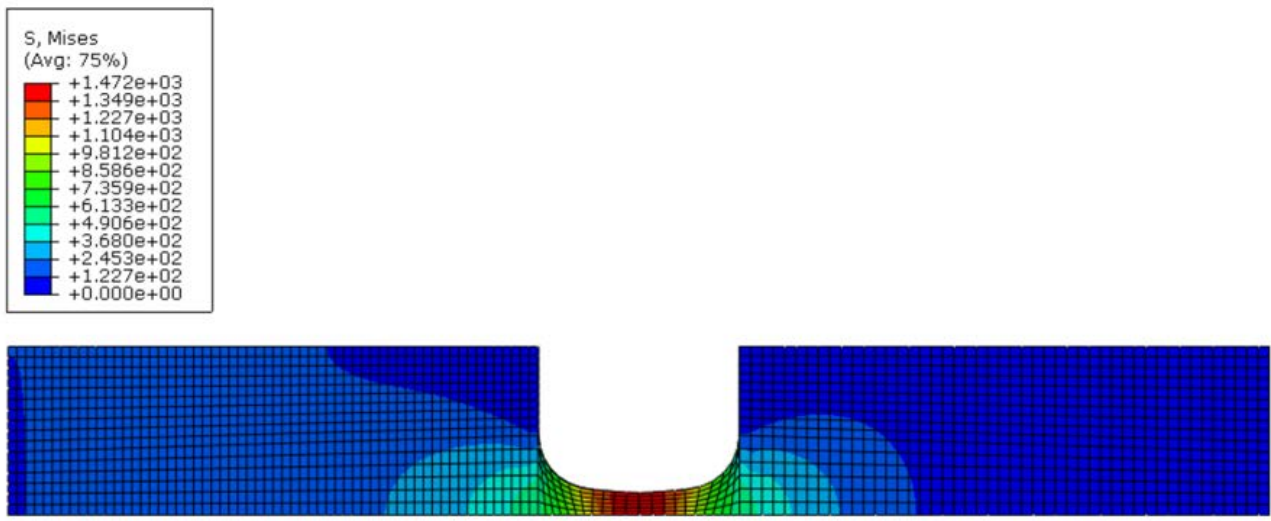

Figure 10: The stress distribution diagram of the specimen at $t_{0}=1.96 \times 10^{-4} \mathrm{~s}$. 


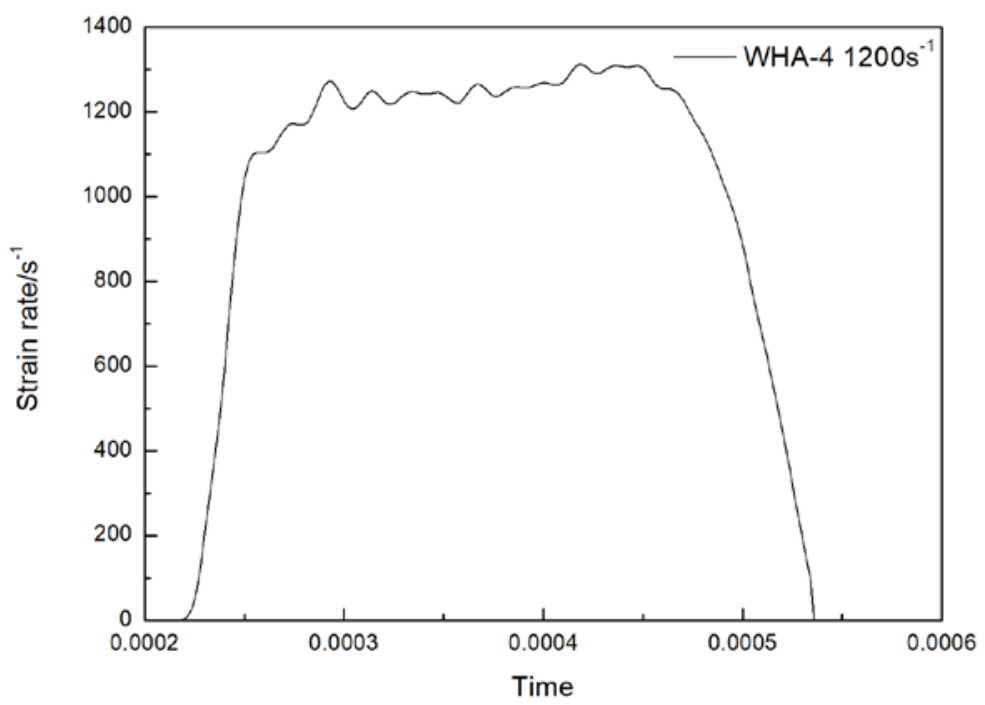

Figure 11: Strain rate curve in simulation under $1200 \mathrm{~s}^{-1}$

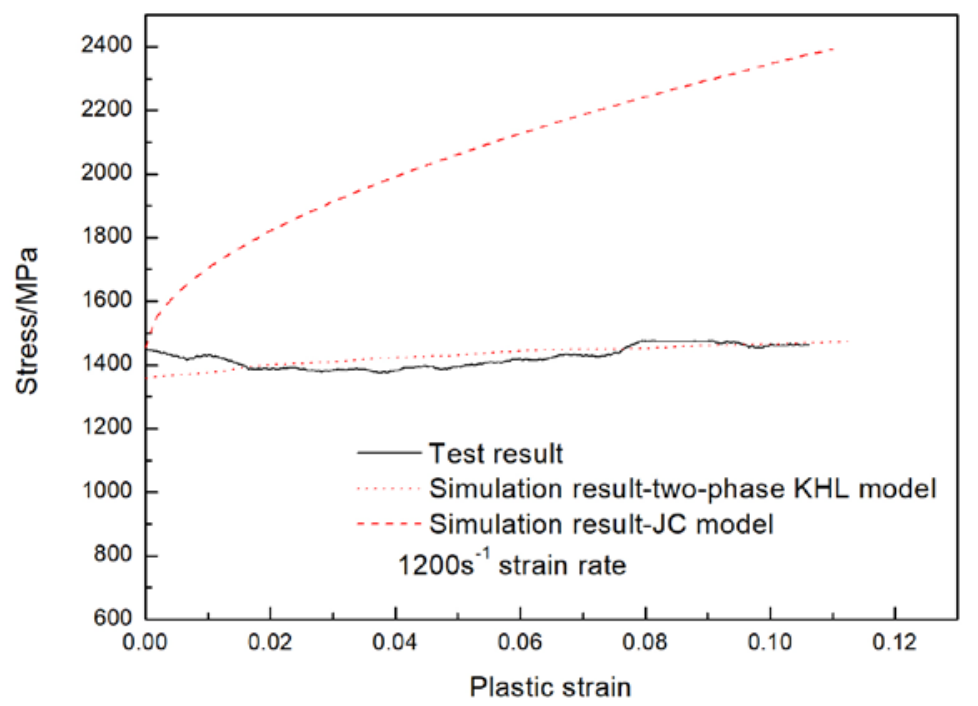

Figure 12: Stress-plastic strain curve of $1200 s^{-1}$ strain rate

The comparison of simulation results and test results of $92.5 \%$ tungsten heavy alloy under other working conditions are shown in Figure 13. It can be seen that the simulation results of the proposed constitutive model for different strain rates are in good agreement with the test results, except for some cases of high strain rate conditions. The strain softening effect of $92.5 \%$ tungsten heavy alloy at high strain rate is obvious, while the influence of strain softening effect has not been well considered in this model. 


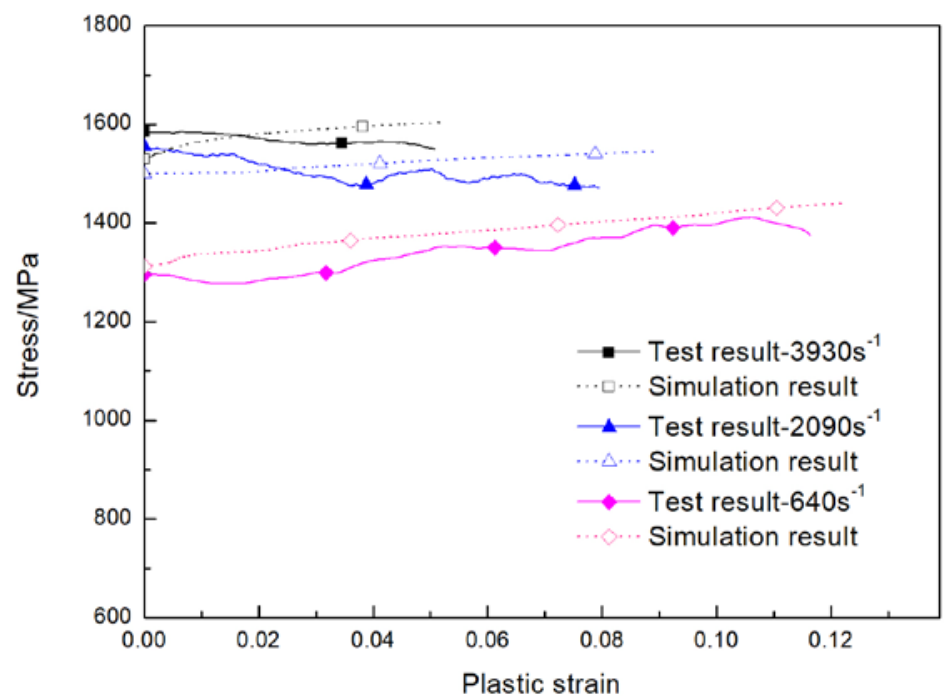

Figure 13: Stress-plastic strain curve of different strain rates

\section{CONCLUSIONS}

In this paper, the static and dynamic mechanical properties of $92.5 \mathrm{~W}-4.9 \mathrm{Ni}-2.1 \mathrm{Fe}-0.5 \mathrm{Co}$ are studied by tests. Based on mesomechanics theory, the property and the stress-strain relationship of each phase are studied by combining with Eshelby equivalent inclusion theory and Mori-Tanaka mean field theory. Then the damage variable is introduced according to the continuous damage mechanics theory, and a two-phase constitutive model of tungsten heavy alloy with damage is established by combining damage evolution model with constitutive model. Then, the finite element simulations for 92.5\% tungsten heavy alloy are carried out. The simulation results are in good agreement with the test results, which verifies the effectiveness of the proposed model. The main conclusions in this paper are as follows:

1) The results of tensile property tests show that the strain hardening effect is obvious in the static stress-strain curve of $92.5 \%$ tungsten heavy alloy. With the increase of strain rate, the strain hardening effect gradually weakens, and even the strain softening effect occurs at high strain rate. Therefore, the $92.5 \%$ tungsten heavy alloy is suitable for KHL constitutive model.

2) The results show that the mechanical properties of tungsten phase and binder phase of 92.5\% tungsten heavy alloy are quite different. The tungsten phase has high strength but poor plasticity, while the binder phase has low strength but good plasticity. Therefore, the stress-strain state and damage condition of the two phases are different. The damage evolution in the two phases affects the mechanical properties of the alloy, respectively.

3) Considering the accumulation of damage evolution and that the stress-strain state needs to be updated according to the previous damage value, the finite element simulation of the constitutive model is implemented with UMAT, and the simulation results are in good agreement with the test results. 


\section{REFERENCES}

[1] T. Liu, J. L. Fan B. Y. Huang, M. G. Qi and J. M. Tian. "Effect of Mechanical alloying and trace $\mathrm{Y}_{2} \mathrm{O}_{3}$ addition on microstructure of fine-grain tungsten heavy alloy rods," Rare Metal Materials and Engineering, 39(2), 314-317(2010).

[2] Z. H. Zhang, F. C. Wang, S. K. Li and L. Wang. "Deformation characteristics of the 93W-4.9Ni-2.1Fe tungsten heavy alloy deformed by hydrostatic extrusion," Materials Science \& Engineering A, 435, 632-637(2006).

[3] S. Humail, F. Akhtar, S. J. Askari, M. Tufail and X. Qu. "Tensile behavior change depending on the varying tungsten content of W-Ni-Fe alloys," International Journal of Refractory Metals and Hard Materials, 25(5-6), 380-385 (2007).

[4] R. Gero, L. Borukhin and I. Pikus. "Some structural effects of plastic deformation on tungsten heavy metal alloys,” Materials Science \& Engineering A, 302(1), 162167(2001).

[5] J. Das, G. A. Rao and S. K. Pabi. "Microstructure and mechanical properties of tungsten heavy alloys,” Materials Science \& Engineering A, 527(29-30), 7841-7847(2010).

[6] R. M. Luo, D. W. Huang, M. C. Yang, E. L. Tang, M. Wang and L. P. He. "Penetrating performance and "self-sharpening" behavior of fine-grained tungsten heavy alloy rod penetrators," Materials Science \& Engineering A, 675, 262-270(2016).

[7] G. R. Johnson and W. H. Cook. "A constitutive model and data for metals subjected to large strains, high strain rates and high temperatures," Engineering Fracture Mechanics 21, 541-548(1983).

[8] S. Khan and R. Liang. "Behaviors of three BCC metal over a wide range of strain rates and temperatures: experiments and modeling,” International Journal of Plasticity, 15(10), 1089-1109(1999).

[9] S. Khan, Y. S. Suh and R. Kazmi. "Quasi-static and dynamic loading responses and constitutive modeling of titanium alloys," International Journal of Plasticity, 20(12), 2233-2248(2004).

[10]F. J. Zerilli and R. W. Armstrong. "Dislocation-mechanics-based constitutive relations for material dynamics calculations,” Journal of Applied Physics, 61(5), 1816-1825(1987).

[11]F. J. Zerilli and R. W. Armstrong. "Description of tantalum deformation behavior by dislocation mechanics based constitutive relations," Journal of Applied Physics, 68(4), 1580-1591(1990).

[12]F. J. Zerilli and R. W. Armstrong. "The effect of dislocation drag on the stress-strain behavior of F.C.C. metals,” Acta Metallurgica Et Materialia, 40(8), 1803-1808(1992).

[13]J. Klepaczko. "Thermally activated flow and strain rate history effects for some polycrystalline F.C.C. metals,” Materials Science \& Engineering, 18(1), 121-135(1975).

[14] P. S. Follansbee and U. F. Kocks. "A constitutive description of the deformation of copper based on the use of the mechanical threshold stress as an internal state variable." Acta Metallurgica, 36, 81-93(1988). 
[15] D. J. Steinberg, S. G. Cochran, and M. W. Guinan. "A constitutive model for metals applicable at high-strain rate,” Journal of Applied Physics, 51(3), 1498-1504(1980).

[16] X. Gong, J. Fan and F. Ding. "Tensile mechanical properties and fracture behavior of tungsten heavy alloys at $25-1100{ }^{\circ} \mathrm{C}$," Materials Science \& Engineering A, 646, 315321(2015).

[17] G. R. Johnson and W. H. Cook. "Fracture characteristics of three metals subjected to various strains, strain rates, temperatures and pressures," Engineering Fracture Mechanics, 21(1), 31-48(1985).

[18] S. Murakami. “Continuum damage mechanics,” Netherlands: Springer(2012).

[19] J. D. Eshelby. Proceedings of the Royal Society, A241, 376-396(1957).

[20] T. Mori, and K. Tanaka. "Average stress in matrix and average elastic energy of materials with misfitting inclusions,” Acta Metallurgica, 21(5), 571-574(1973).

[21] L. L. Wang, X. L. Dong and Z. J. Sun. "Dynamic constitutive behavior of materials at high strain rate taking account of damage evolution,” Explosion and Shock Waves, 26, 193-198(2006).

[22] Y. Z. Ma, W. S. Liu, and P. H. He. "Effects of Co addition on microstructure and properties of tungsten-based alloys by solid-solution and water-quenching," The Chinese Journal of Nonferrous Metals, 21(5), 1016-1020(2011).

[23] N. Senthilnathan, A. R. Annamalai and G. Venkatachalam. "Microstructure and mechanical properties of spark plasma sintered tungsten heavy alloys," Materials Science \& Engineering A, 710, 66-73(2017).

[24] F. Y. Lu and R. Chen, “Hopkinson Bar Techniques,” Science Press (2013).

[25] W. S. Lee, G. L. Xiea and C. F. Lin. "The strain rate and temperature dependence of the dynamic impact response of tungsten composite,” Materials Science \& Engineering A, 257(2), 256-267(1998).

[26] Q. S. Chen, Y. G. Miao, Y. Z. Guo and Y. L. Li. Chinese Journal of High Pressure Physics, 31(6), 753-760(2017).

[27] G. Prabhu, N. A. Kumar, M. Sankaranarayana and T. K. Nandy. "Tensile and impact properties of microwave sintered tungsten heavy alloys," Materials Science \& Engineering A, 607, 63-70(2014).

[28] C. C. Zhu, Y. T. Song, X. B. Peng, Y. P. Wei, X. Mao, W. X. Li and X. Y. Qian. "The dynamical mechanical properties of tungsten under compression at working temperature range of divertors,” Journal of Nuclear Materials, 469, 120-124(2016).

[29] J. Lemaitre. “A course on damage mechanics,” Springer-Verlag (1996).

[30] X. H. Liu, X. C. Huang, Y. Z. Chen, X. Y. Su and J. S. Zhu. “A review on constitutive models for plastic deformation of metal materials under dynamic loading," Advances in Mechanics, 37(3), 361-374(2007).

[31] H. L. Wan, Q. Z. Wang, C. X. Jia and Z. Zhang. "Multi-scale damage mechanics method for fatigue life prediction of additive manufacture structures of Ti-6Al-4V," Materials Science \& Engineering A, 669, 269-278(2016).

[32] Belytschko, T., et al. [2014] "Nonlinear finite elements for continua and structures," John Wiley \& Sons Inc. 
